\title{
Microstructural Dynamics of the Myocardium: Orientation of the Muscle Fibers and Occurrence of Cardiomyopathies
}

\author{
René Thierry Djoumessi iD and François Beceau Pelap $i$ \\ UR de Mécanique et de Modélisation des Systèmes Physiques (UR-2MSP), Faculté des Sciences, Université de Dschang, \\ BP 69 Dschang, Dschang, Cameroon
}

Correspondence should be addressed to François Beceau Pelap; fbpelap@yahoo.fr

Received 5 October 2021; Accepted 20 December 2021; Published 12 January 2022

Academic Editor: Guosong Wu

Copyright ( 92022 René Thierry Djoumessi and François Beceau Pelap. This is an open access article distributed under the Creative Commons Attribution License, which permits unrestricted use, distribution, and reproduction in any medium, provided the original work is properly cited.

\begin{abstract}
This paper considers the Holzapfel-Ogden (HO) model to examine the behavior of the left ventricle myocardium. At the tissue level, we analyze the contributions of the orientation angle of muscle fibers (MFs) and investigate their effects on the occurrence of certain cardiomyopathies and congenital diseases at the organ level. Knowing the importance of myocardial microstructure on cardiac function, we vary the angle between the direction of collagen sheets and MFs in all layers of the myocardium (from epicardium to endocardium) to model the effects of tilted MFs. Based on the HO model in which the directions of the fibers are orthogonal and using the strain energy of HO, we construct a tensile-compression test and simulate the dynamics of a cubic sample. We recover the authors' results exhibiting the existence of residual stresses in various directions. Then, we modify the energy of HO slightly to assess the impact of the same stress states on the system with tilted MFs. A numerical tensile-compression test performed on this new cubic sample shows that, in certain directions, the heart tissue is more resistant to shear deformations in some planes than in others. Moreover, it appears that the residual stress is smaller as the angle of orientation of the MFs is small. Furthermore, we observe that the residual stress is greater in the new model compared to the normal HO model. This could affect the heart muscle at the organ level leading to hypertrophied/dilated cardiomyopathy.
\end{abstract}

\section{Introduction}

Several works have been carried out to understand the electromechanical behavior of the heart $[1,2]$. This modeling requires knowledge of the morphology of the heart [3-5], its electrical behavior [6], and its mechanical behavior [3, 7]. However, the mechanical behavior of the heart remains a mystery due to the difficulty of finding a stress-strain relationship. Fortunately, several studies have already been performed to determine the mechanical properties and structure of heart tissue. Holzapfel and Ogden [8] proposed a nonlinear law describing its dynamics and revealing the existence of residual stresses in various directions. As muscle fibers (MFs) and collagen sheets' matrices make up heart tissue, $\mathrm{HO}$ exploited this structuring but assumed that MFs are perpendicular at the reference configuration (RC); hypothesis neglects the contribution of the orientation angle of
MFs at the RC. Fortunately, there are some works which take into account the orientation of MFs.

In blood vessels, Agianniotis et al. [9] demonstrated that the structural model of the vascular wall is less sensitive to orientation dispersion when the fibers are mainly oriented circumferentially. Cyron and Humphrey [10] analyzed the same behavior and established that some orientation of the fibers reinforces the structure of the artery. In electrophysiological models, Baier et al. [11] used MFs' orientation to construct the diffusion tensor for electrical potential propagation. However, other researchers proved that despite the collagen fibers are tilted at the organ level, the directions of MFs and collagen sheets remain perpendicular at the local level [12] HO [8]. In fact, at the local level, the myocardial microstructure tissue is composed of layers.

On the contrary, the tilt of MFs is observed in the tissues of patients with congenital diseases $[13,14]$. This is usually 
seen in children at birth. This inclination appears during the development of the heart which leaves from a cylindrical cavity to a cavity with four chambers by undergoing irreversible poor morphogenesis [15]. For the elderly, tilting of MFs usually occurs after surgery [16]. However, thinking that the inclination of fibers at the level of the tissues may be the cause of certain heart diseases or the occurrence of certain congenital diseases is not yet well developed in the literature.

Motivated by the aforementioned interests in collagen fibers in various fields, we believe that considering the orientation angle of MFs in heart tissue could lead to new features regarding its fibrous structure and mechanical behavior. In this paper, a technique to better explain the mechanism of occurrence of certain congenital diseases is proposed. Then, we investigate the impact of the inclination of MFs on the mechanical behavior of the left ventricle. The outcome of this study is structured as follows. In Section 2, we introduce a tensile-compression test and simulate the dynamics of a small cubic sample of tissue $\left(10 \times 10 \times 1 \mathrm{~mm}^{3}\right)$ in which the MFs are not tilted, describing the $\mathrm{HO}$ model. Next, we design the modified Holzapfel-Ogden (MHO) model dealing with inclined MFs and new strain energy. The simplifying hypotheses and the mathematical formulation are presented. In addition, the stress variation with respect to the tilt angle of the MFs is examined. In Section 3, various established results with corresponding comments are exposed. Discussion and concluding remarks are given in Section 4.

\section{Materials and Methods}

2.1. Simplifying Hypotheses. In this study, we formulate the upcoming assumptions. (i) We focus our attention on the myocardium of the left ventricle and consider it as nearly incompressible and less viscous [17]. (ii) The passive myocardium tissue is an orthotropic material having three mutually orthogonal planes with distinct material responses. (iii) The angle of orientation of MFs changes in the myocardium from the epicardium to the endocardium. However, it is possible to associate an orthogonal reference to the direction of the fibers. Therefore, no matter which layer is located between epicardium and endocardium, this reference remains valid. (iv) To model the poor organization of MFs at the microstructural level, we presume that, on each layer, the angle between the direction of MFs and that of collagen sheets is not $90^{\circ}$ but varies very slightly. $(v)$ We consider that all the phenomena which can occur at the level of the tissues could be reproduced at the level of the organs. Due to the complexity of MFs at the organ level and the difficulty of associating a reference with it, we will work at the local level and try to predict what might happen at the organ level.

The mathematical approach utilized is a semianalytical method which aims to highlight the stresses in the different planes of the tissue at the local level. Moreover, the analyses made here are based on an explanation of the stresses generated in the muscle during our simulations.
2.2. Model of the Myocardium. We consider the cardiac muscle model proposed by HO (2009) and depict in Figure 1 . The parameters $f_{0}, s_{0}$, and $n_{0}$ represent, respectively, the unit vectors in the direction of the fibers, the collagen sheets, and the normal to the plane formed by the two preceding directions. Due to the complexity of linking myocardium to a simple reference frame, it is admitted that the three abovementioned directions constitute its unit coordinates.

In the reference configuration, HO (2009) considered that directions of the MFs are perpendicular. In this work, we exploit their nonlinear model and assume that MFs are inclined of $\alpha$ to reflect the fact that the cardiac muscle has undergone a deformation (Figure 2). Note that $\alpha$ is the angle between the collagen sheet and the directions of the muscle fibers. Now, the unit vectors are defined as

$$
\begin{aligned}
& f_{0}\left(\begin{array}{c}
\cos \alpha \\
\sin \alpha \\
0
\end{array}\right), \\
& s_{0}\left(\begin{array}{l}
0 \\
1 \\
0
\end{array}\right), \\
& n_{0}\left(\begin{array}{l}
0 \\
0 \\
1
\end{array}\right) .
\end{aligned}
$$

The idea behind the parameter $\alpha$ is to suggest a mathematical model of the cardiac muscle with a congenital disease or a form of cardiomyopathy and examine its dynamics. The limit case $\alpha=0$ yields the $\mathrm{HO}$ results.

2.3. Mathematical Formulation. Let $\Omega_{0}$ and $\Omega$ be the nondeformed and deformed configurations of the myocardium. The quantities $X_{i}$ and $x_{i}$ denote, respectively, the coordinates bound to $\Omega_{0}$ and $\Omega$ such that the transformation to leave the reference configuration $\left(\Omega_{0}, X_{i}\right)$ to the current configuration $\left(\Omega, x_{i}\right)$ is defined by $x_{i}=\varphi\left(X_{i}\right)$, where $\varphi$ is the transformation function. The strain gradient tensor allowing describing the local kinematics of the system is

$$
F_{i, j}=\frac{\partial \varphi_{i}}{\partial X_{j}}, \quad \text { with } i, j \in\{1,2,3\} .
$$

We associate this tensor to the left and right CauchyGreen stress tensors and to the Green-Lagrange strain tensor which are, respectively,

$$
\begin{aligned}
& C=F^{T} F, \\
& B=F F^{T}, \\
& E=\frac{1}{2}(C-I) .
\end{aligned}
$$

The strain energy of HO (2009) is exploited to define these invariants: 


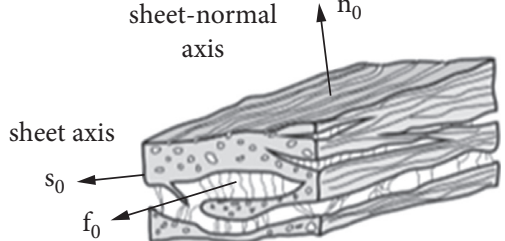

fibre axis

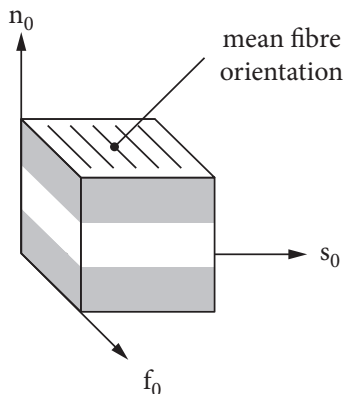

$f_{0}$

FIgURE 1: HO model (2009) showing the microscopic section of the heart muscle.

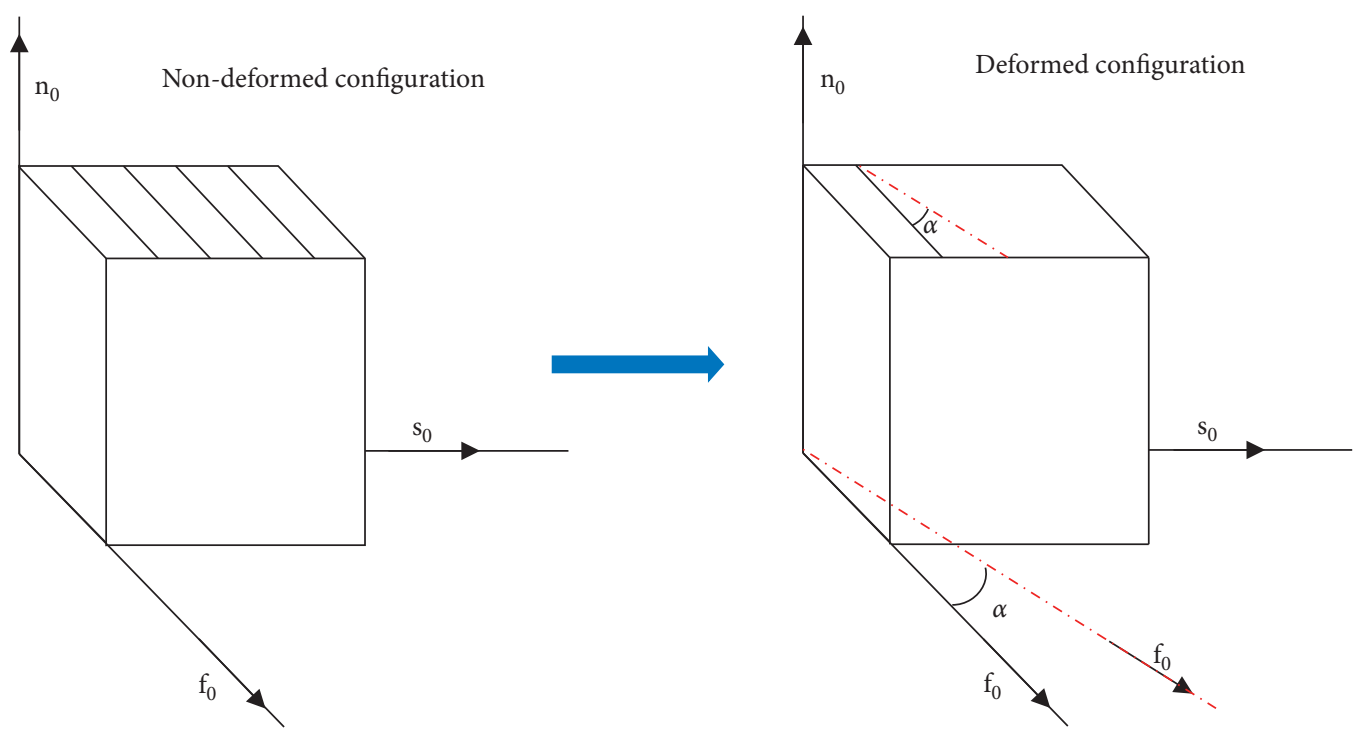

Figure 2: Proposed modified HO nodel exhibiting the microscopic section of the heart muscle with MFs inclined of an angle $\alpha$.

$$
\begin{aligned}
& \left\{\begin{array}{l}
I_{1}=\operatorname{trace}(C), \\
I_{4 f}=f_{0} \cdot\left(C f_{0}\right),
\end{array}\right. \\
& \left\{\begin{array}{l}
I_{4 s}=s_{0} \cdot\left(C s_{0}\right), \\
I_{8 f s}=f_{0} \cdot\left(C s_{0}\right)=s_{0} \cdot\left(C f_{0}\right) .
\end{array}\right.
\end{aligned}
$$

The stress tensor derives from the potential (strain energy) such as

$$
\begin{aligned}
\sigma_{p} & =F \frac{\partial \Psi}{\partial F} \\
& =F \frac{\partial \Psi}{\partial E} F^{T}, \\
S_{p} & =2 \frac{\partial \Psi}{\partial E}
\end{aligned}
$$

where $\sigma_{p}$ and $S_{p}$ denote, respectively, the Cauchy stress tensor and the second Piola stress tensor for case of passive myocardium. The quantity $\Psi$ designates the deformation energy:

$$
\begin{aligned}
\Psi= & \frac{a}{2 b} \exp \left[b\left(I_{1}-3\right)\right]+\sum_{i=f, s} \frac{a_{i}}{2 b_{i}}\left\{\exp \left[b_{i}\left(I_{4 i}-1\right)^{2}\right]-1\right\} \\
& +\frac{a_{f s}}{2 b_{f s}}\left[\exp \left(b_{f s} I_{8 f s}^{2}\right)-1\right] .
\end{aligned}
$$

In (6), factors $a, b, b_{i}, a_{i}, a_{f s}$, and $b_{f s}$ deal with the properties of the material presented in Table 1, with $i \in\{f, s\}$. Therefore, the passive stress tensor of Cauchy $\sigma_{p}$ becomes

$\sigma_{p}=2 \Psi_{1} B+2 \Psi_{4 f o} \cdot f \otimes f+2 \Psi_{4 s o} \cdot s \otimes s+2 \Psi_{8 f s} \cdot(f \otimes s+s \otimes f)$,

where $\Psi_{1}, \Psi_{4 f o}, \Psi_{4 s o}$, and $\Psi_{8 f s}$ represent the strain energies induced, respectively, by the invariants of the tensors $I_{1}, I_{4 f}$, $I_{4 s}$, and $I_{8 f s}$, that is, 
TABLE 1: Values of the system parameters.

\begin{tabular}{lcccccccc}
\hline Parameters & $a$ & $b$ & $a_{f}$ & $b_{f}$ & $a_{s}$ & $b_{s}$ & $a_{f s}$ & $b_{f s}$ \\
\hline Values & $0.059 \mathrm{kPa}$ & 8.023 & $18.472 \mathrm{kPa}$ & 16.026 & $2.481 \mathrm{kPa}$ & 11.120 & 0.216 & $11.43 \mathrm{kPa}$ \\
\hline
\end{tabular}

$$
\begin{gathered}
\left\{\begin{array}{l}
f=F f_{0}, \\
s=F s_{0},
\end{array}\right. \\
\text { with }\left\{\begin{array}{l}
\Psi_{1}=\frac{\partial \Psi}{\partial I_{1}}, \\
\Psi_{4 f o}=\frac{\partial \Psi}{\partial I_{4 f}},
\end{array}\right. \\
\left\{\begin{array}{l}
\Psi_{4 s o}=\frac{\partial \Psi}{\partial I_{4 s}}, \\
\Psi_{8 f s}=\frac{\partial \Psi}{\partial I_{8 f s}} .
\end{array}\right.
\end{gathered}
$$

In the RC, we start with the modified model of Figure 2 and examine its dynamics by performing a shear in the $f s$ plane, more precisely in the $s_{0}$ direction. In this shear mode, MFs are stretched and the corresponding tensor strain gradient is

$$
[F]=\left[\begin{array}{lll}
1 & 0 & 0 \\
\lambda & 1 & 0 \\
0 & 0 & 1
\end{array}\right]
$$

where $\lambda$ defines the stretch in collagen sheets direction. For the shear in $s_{0}$ direction, we have

$$
\begin{aligned}
{[B] } & =\left[\begin{array}{ccc}
1 & \lambda & 0 \\
\lambda & 1+\lambda^{2} & 0 \\
0 & 0 & 1
\end{array}\right], \\
f & =f_{0}+\lambda, \\
s_{0} s & =s_{0}, \\
n & =n_{0} .
\end{aligned}
$$

The analytical expressions of the stresses according to the strain energy and the strain field used are expressed below:

$$
\sigma_{f f}=2\left(\lambda^{2}+1\right) \Psi_{1}+2 \Psi_{4 f o}(\cos \alpha+\lambda \sin \alpha)^{2}+2 \lambda^{2} \Psi_{4 s o},
$$

$$
\begin{aligned}
\sigma_{f s}= & \sigma_{s f} \\
= & 2 \lambda^{2} \Psi_{1}+2 \Psi_{4 f o}[(\cos \alpha+\lambda \sin \alpha) \sin \alpha]+2 \lambda \Psi_{4 s o} \\
& +\Psi_{8 f s}(\cos \alpha+\lambda \sin \alpha),
\end{aligned}
$$

$$
\begin{aligned}
\sigma_{s s} & =2 \Psi_{1}+2 \Psi_{4 f o}(\sin \alpha)^{2}+2 \Psi_{4 s o}+2 \Psi_{8 f s} \sin \alpha, \\
\sigma_{n n} & =2 \Psi_{1},
\end{aligned}
$$

in which

$$
\begin{gathered}
\Psi_{1}=\frac{a}{2} e^{b\left(I_{1}-3\right)}-\frac{a}{2}, \\
\Psi_{4 f o}=a_{f}\left(I_{4 f}-1\right) e^{b_{f}\left(I_{4 f}-1\right)^{2},} \\
\Psi_{4 s o}=a_{s}\left(I_{4 s}-1\right) e^{b_{s}\left(I_{4 s}-1\right)^{2}}, \\
\Psi_{8 f s}=a_{f s} I_{8 f s} e^{b_{f s} I_{8 f s}^{2}} .
\end{gathered}
$$

Numerical and experimental studies have revealed that the cardiac cycle is composed of an isochoric contraction (at constant volume) and an isovolumic contraction [12]. During the isochoric phase, the muscle is contracted linearly and can be modeled as an isotropic material. Then, the isotropic part $\Psi_{\text {iso }}=-a\left(I_{1}-3\right) / 2$ is introduced into the strain energy. This term aims canceling the residual stress in the $\mathrm{HO}$ model at the RC and may permit to understand impacts of the tilted angle of MFs. Such an expression was first used by Lafortune et al. [7] to force the material to respect the initial conditions but has not been exploited in combination with the complex strain energy function as done in this work.

\section{Results and Discussion}

3.1. Validation of the Numerical Model. To validate our proposed tensile-compression test approach, we consider the myocardium with noninclined MFs $(\alpha=0)$ and carry out numerical simulations for the strain energy established by HO (2009). Figures 3(a)-3(c) exhibit (HO case) the existence of nonneglected residual stresses in privileged directions. These observations are normal because the cardiac tissue possesses residual stresses beforehand. Our numerical code allows recovering results of HO (2009). Therefore, it can be exploited for further investigations.

To better appreciate the effects of the orientation angle of MFs on the occurrence of residual stresses in the cardiac tissue, these constraints should be null in the HO model for $\alpha=0$. To achieve this aim, the isotropic part $\Psi_{\text {iso }}$ is inserted into (6) which becomes

$$
\begin{aligned}
\Psi= & \frac{a}{2 b} \exp \left[b\left(I_{1}-3\right)\right]+\sum_{i=f, s} \frac{a_{i}}{2 b_{i}}\left\{\exp \left[b_{i}\left(I_{4 i}-1\right)^{2}\right]-1\right\} \\
& +\frac{a_{f s}}{2 b_{f s}}\left[\exp \left(b_{f s} I_{8 f s}^{2}\right)-1\right]-\frac{a}{2}\left(I_{1}-3\right) .
\end{aligned}
$$



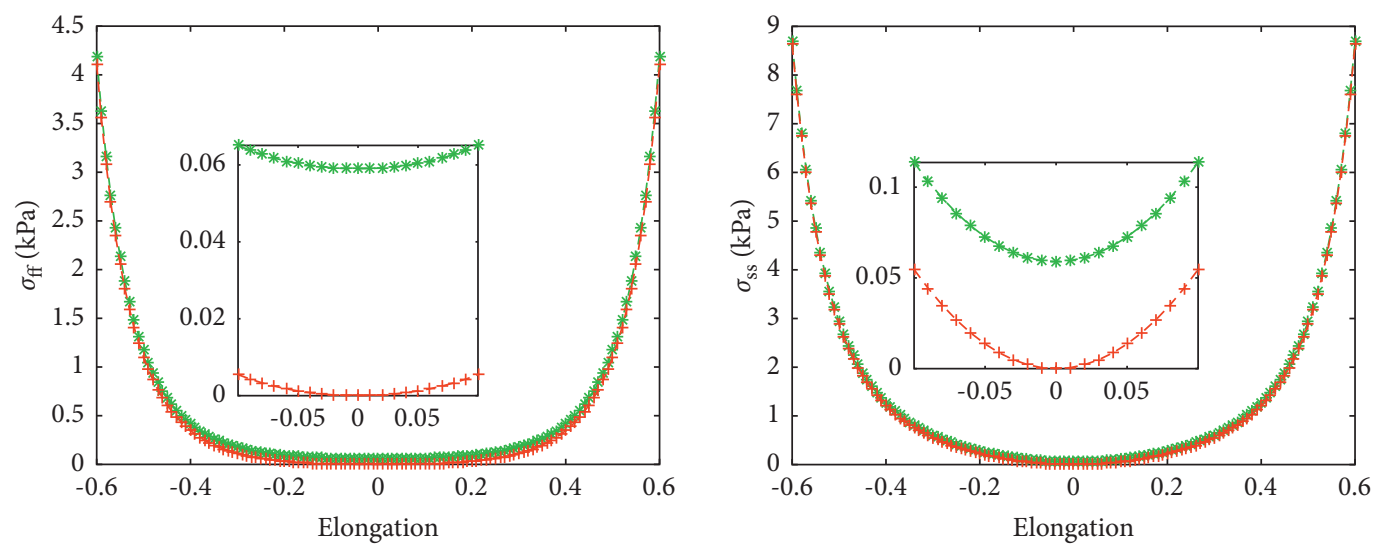

- * - Holzaphel and Ogden Model
-+- HO model with $\Psi_{\text {iso }}$

(a)

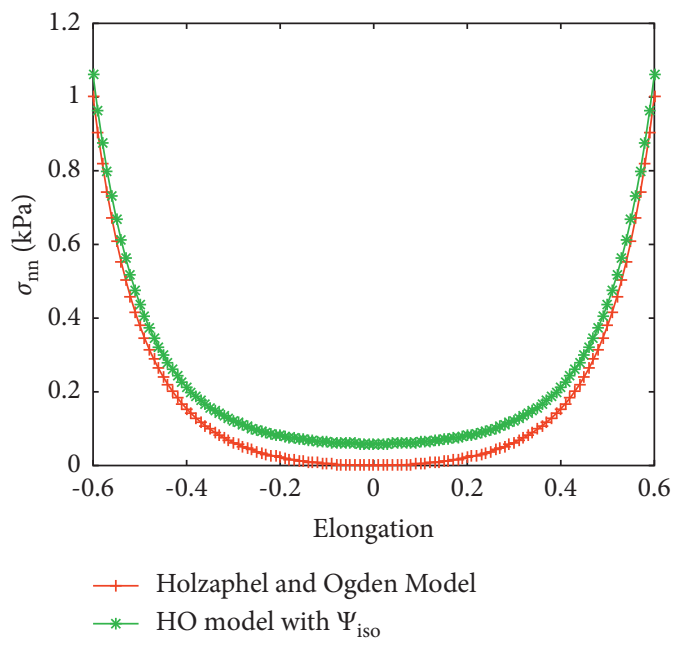

(c)
- * - Holzaphel and Ogden Model
- + - HO model with $\Psi_{\text {iso }}$

(b)

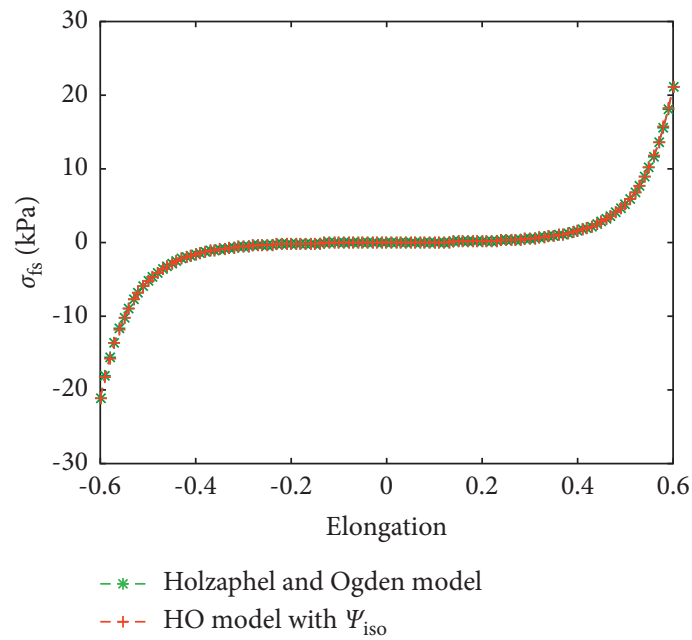

(d)

FIGURE 3: Stress comparison in the directions $f, s, n$, and $f s$ for the HO model and its modified version that includes the isochoric part of the strain energy. These curves display the evolution of the stress in terms of the elongation in the direction of the fibers (a), collagen sheet direction (b), normal direction (c), and fiber-collagen sheet direction (d).

Expression (13) represents the new strain energy of the modified HO model for both $\alpha=0$ and $\alpha \neq 0$ cases.

Figure 3 illustrates the evolution of the Cauchy constraints in the directions $f, s, f_{s}$, and $n$ in terms of the elongation. In these figures, we superimpose the constraints applied on the HO model and those of the HO

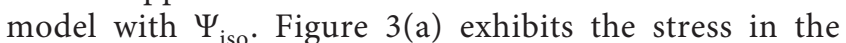
direction of the fiber and shows that the isotropic part affects this stress by setting it to zero. For positive values of the elongation, it appears that the stress increases as the elongation grows and decreases otherwise. The same behaviors are observed in Figures 3(b) and 3(c) which display, respectively, evolutions of the stress in the collagen and collagen-normal directions. However, Figure 3(d) exhibits no significant difference in the combined fiber-sheet direction, since the isotropic part contributes only in both fiber and collagen sheet directions due to the first invariant of the right Cauchy stress tensor.
To summarize, the curves of Figure 3 clearly explain that the $\mathrm{HO}$ model possesses residual stresses. In other words, when the elongation equals zero (naturally at $\mathrm{RC}$ ), one has a significant stress in the $\mathrm{HO}$ model as displayed in Table 2 which perfectly match the observation made in the scientific literature. Likewise, we define a new strain energy (13) which makes it possible to annihilate these stresses in RC. These results will permit us to examine the MHO model without residual stresses in the RC.

\subsection{Contribution of the Tilted Angle of the MFs}

3.2.1. General Results. Based on the MHO model (Figure 2), we deeply investigate the effects of the orientation angle of MFs and the growth of the constraints on the behavior of the myocardium tissue. Therefore, we discuss the occurrence of some diseases (such as cardiomyopathies) linking with the expansion of constraints in the heart muscle. Indeed, cardiomyopathies are known to modify the structure of the tissue and affect constraints by hardening or stretching the 
TABLE 2: Residual stress.

\begin{tabular}{lcc}
\hline Types of stress & HO model (2009) & HO model with $\Psi_{\text {iso }}$ \\
\hline$\sigma_{f f}=\sigma_{s s}=\sigma_{n n}$ & $0.059 \mathrm{kPa}$ & $0.000 \mathrm{kPa}$ \\
$\sigma_{f s}$ & $0.000 \mathrm{kPa}$ & $0.000 \mathrm{kPa}$ \\
\hline
\end{tabular}

tissue. This action ultimately increases the stress of the heart muscle [18]. However, our strategy will not allow explaining the occurrence of congenital diseases at birth but will help to predict what might happen and how certain congenital diseases might develop after birth.

On the contrary, when the MFs are tilted (the MHO model with (13)), the ventricular myocardium seems to be more resistant to shear deformations that produce an extension of the myocyte axis $f$ in the $f s$ plane. Indeed, this resistance is higher in the positive plane for positive angles and zero in the negative plane (Figure 4(b)) and is also greater in the negative plane for negative angles and null in the positive plane (Figure 4(a)). These graphs exhibit the growth of stresses with the tilted angle of the MFs.

Moreover, it appears that, in the positive plane of the $\mathrm{HO}$ model (Figure 3(d)), the stress is lower than that of the corresponding modified model (Figure 4(b)) for positive angles. The same observations are made in the negative plane for negative angles. These results show that the stresses are greater in the MHO model due to the effects of the tilt angle of the MFs. There exist indirect experimental evidences suggesting that the characteristics and extent of the extracellular connective tissue matrix are important determinant of diastolic and systolic ventricular function [19].

Figure 5 exhibits the increase in stresses with growth of tilted angle of the MFs. For positive angles, the constraints are important in the direction of the collagen sheets (Figure 5(b)) for a tensile test. Indeed, when the fibers are inclined with positive angles (counterclockwise), the myocardium is prestressed. For people with such a myocardium and based on the fact that the myocardium is constantly stretched, they may experience blood overload in the ventricles leading to dilated cardiomyopathy (DCM) such as eccentric cardiomyopathy [4]. Since DCM occurs when the heart muscle is too weak to pump blood efficiently, muscles stretch and thin. This allows the chambers of the heart to expand. One observes in Figure 5(a) that the muscle is initially stretched in the direction of the fiber and that it stretches more as the angle of inclination grows. Because the stress in the initial position is not zero, the muscle cannot be contracted directly, justifying the high-stress values obtained in the direction of collagen sheets and in the MFs direction. As the heart chambers are initially dilated, the heart muscle does not contract normally and cannot pump blood very well.

Similar analyses are performed for negative values of the orientation angle of MFs. Indeed, we observe that, for negative angles, the stresses increase as the angle grows, enlarge the cells of the heart muscle, stretch the walls of the ventricles, and cause their thickening. The size of the ventricle does not change significantly and remains near normal, but thickening can block blood flow out of the ventricle [20]. Figure 6 shows the evolution of stress in the direction of the fiber exhibiting the significant stress developed by a muscle to contract. When this happens, the heart undergoes hypertrophic obstructive (concentric) cardiomyopathy [21].

In most cases of cardiomyopathy, the heart muscle weakens and is unable to pump blood to the rest of the body as it should. This work shows that the orientation angle of MFs can cause such cardiomyopathies. As displayed in Figures 5 and 6 , the muscle is already under great stress at $\mathrm{RC}$. This stress prevents the muscle from contracting normally and forces the muscle to develop significant stress to do the same work as a healthy muscle. In fact, the more the angle increases, the more the stress at the RC grows, and consequently, the muscle remains hard or expands according to the sign of the tilted angle.

3.2.2. Impact on the Blood Circulation. Analyzes of the blood circulation aspect reveal (Figure 4) that the MFs orientation angle stretches the heart muscle more in some planes than in others causing degradation of the cardiac cycle. Indeed, after a cycle of contraction-relaxation, the pressure-volume loop is more stretched and flatter [22]. According to Cargo's hypothesis, when the myocardium is stretched, the biaxially organized connective tissue networks from the epimysium to the endomysium reorganize to align more closely with the direction of the MFs. In our model, we consider the pathology by assuming that fibers are tilted leading to a disorganized alignment. This disorganization will impact the cardiac function which directly affects the pressure-volume curve of the ventricle.

Diastolic dysfunction happens when the heart fails to relax completely, so the volume of blood needed by the organs is reduced. This situation is due to an extra-reversible growth characterized by nonzero constraints at the RC. This acute reversibility causes a state of residual stress in the medium which is intrinsic to the shortening of myofibril. For this reason, the heart muscle expands and is no longer under strain from the hemodynamic pressure [15]. These results are in perfect agreement with the findings of Caulfield and Borg [23] who assert that the substantial changes in ventricular wall thickness that occur during the cardiac cycle are only possible due to slippage and rearrangement of the ventricular wall.

3.2.3. Links with Clinical Observations. Clinical observations have established that people with congenital diseases possess heart tissue with tilted MFs (Ghonim et al., 2017). Therefore, the MHO model provides insight into the 


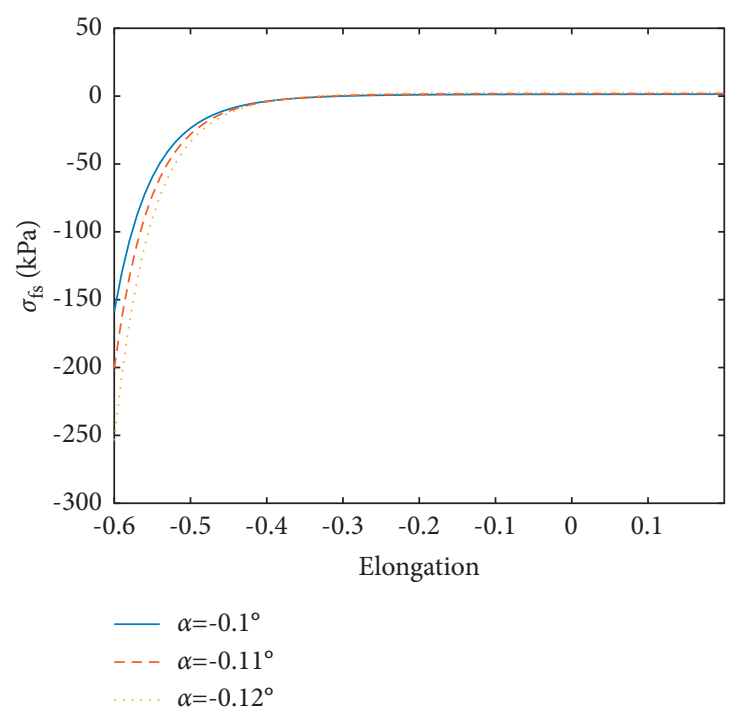

(a)

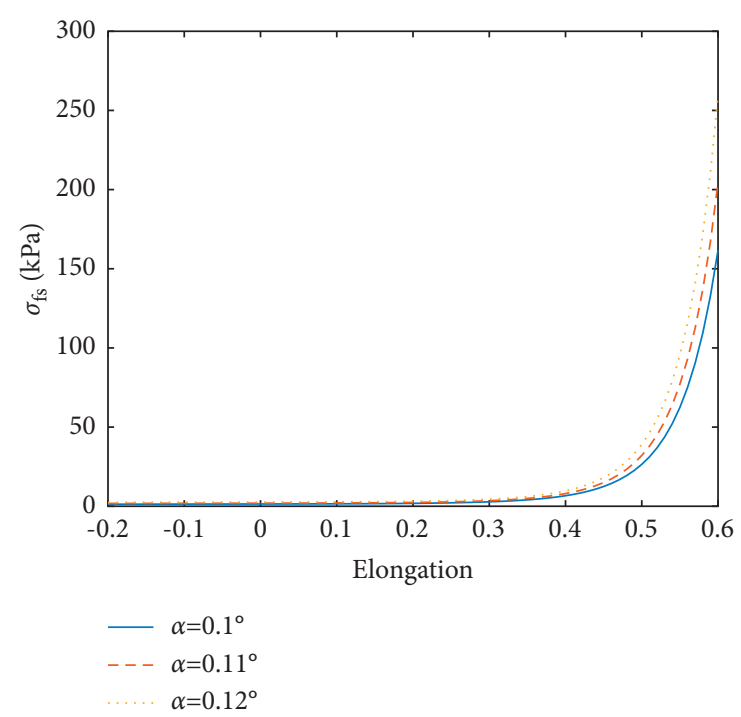

(b)

Figure 4: Behavior of the heart tissue for (a) negative and (b) positive values of the orientation angle of the MFs.

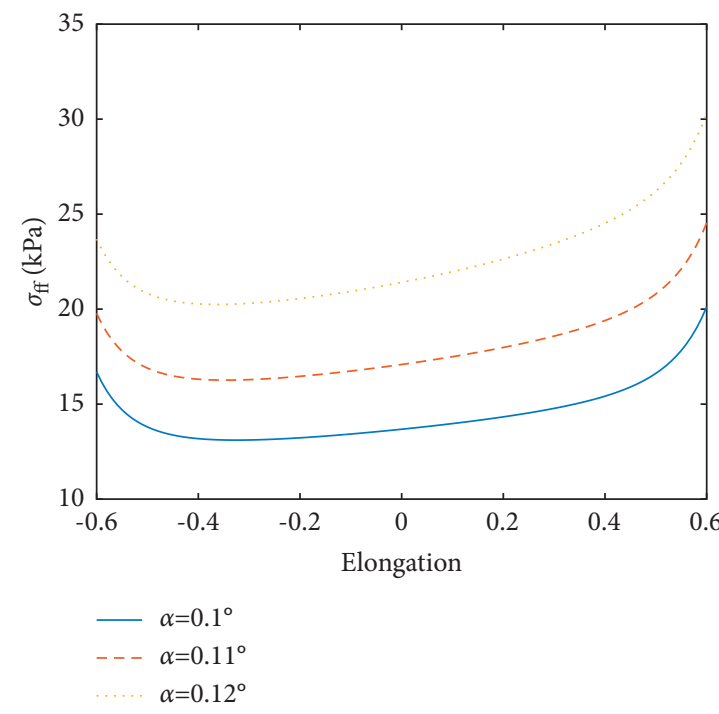

(a)

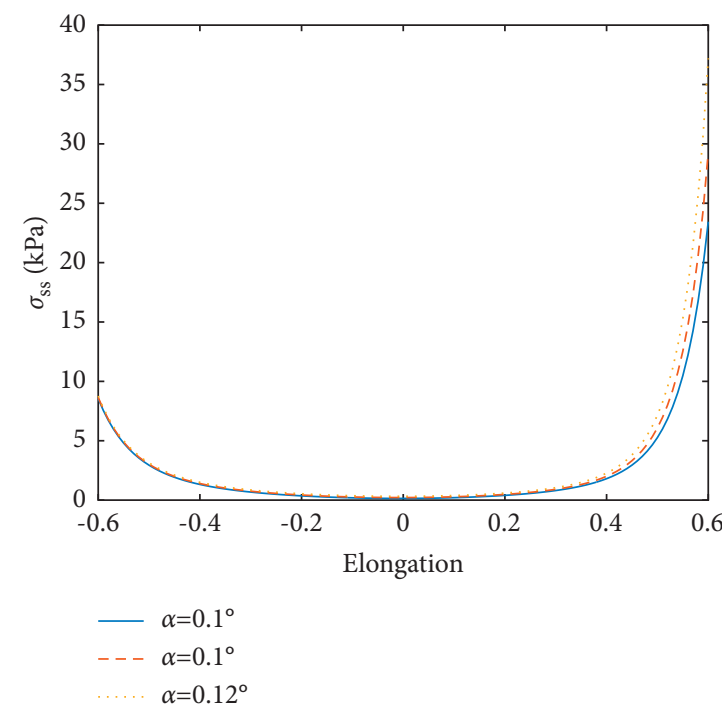

(b)

FIGURE 5: Stress variation versus the elongation in the direction of the fibers (a) and in the direction of the collagen sheets (b) for various orientation angles of the MFs.

stressful state of the heart of a patient with congenital disease. This stressful state may be due to surgery, morphogenesis and/or muscle growth related to poor heart muscle structure. Since the myocardium is an assembly of tissues, observations made at the tissue level could be extended at the organ level. 


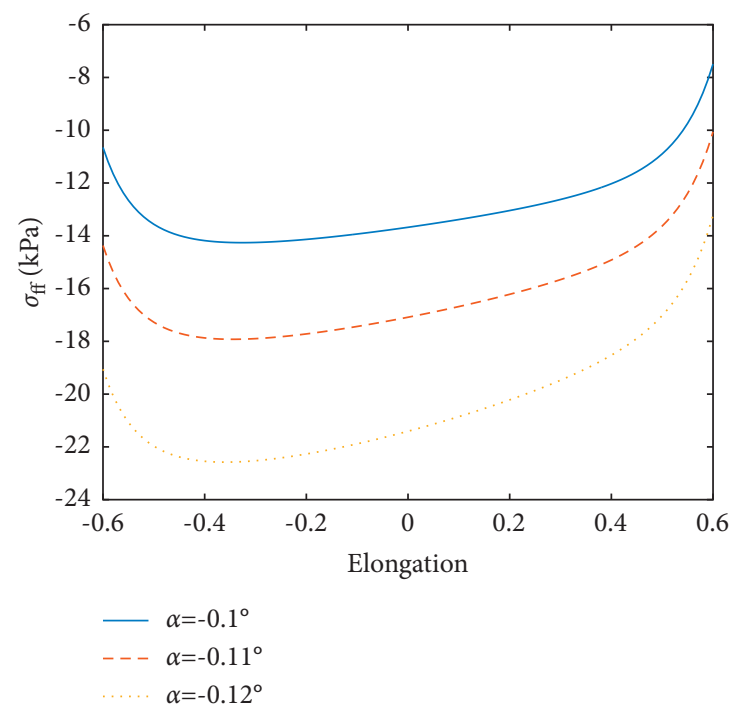

FIgURE 6: Behavior of the heart tissue in the direction of the fibers for negative values.

\section{Conclusion}

In this paper, we investigated the microstructural dynamics of the myocardium and its impact on cardiac functions. We analyzed the effects of the orientation angle of MFs on the behavior of cardiac tissue and on the occurrence of certain forms of cardiomyopathy. Therefore, we performed a numerical tensile-compressive test on a cubic sample of the myocardium defined by $\mathrm{HO}$ (2009) and recovered their results which validate our model. Since the inclination of MFs is observed in several cases of patients with congenital diseases, we introduced at the microstructure level, a modified model in which all the directions of the fibers (collagen sheet, muscular fibers, and normal direction) form a nonorthogonal reference in each layer of the myocardium. The results on this new sample displayed that, in certain directions, the heart tissue is more resistant to shear deformations which produce an extension of the myocyte axis $(f)$ in the plane $(f s)$. It appeared that the stresses increase with the growth in the angle of inclination of the MFs. During comparative studies, we demonstrated that the residual stress is greater in the MHO model than in the HO model. Such residual stress could affect cardiac tissue leading to certain forms of cardiomyopathy. Our results were also compared to existing ones and agreed very well.

\section{Data Availability}

The data used to support the findings of this study are included within the article.

\section{Conflicts of Interest}

The authors declare that they have no conflicts of interest.

\section{References}

[1] H. Xia, K. Wong, and X. Zhao, "A fully coupled model for electromechanics of the heart," Computational and mathematical methods in medicine, vol. 2012, no. 1, 10 pages, Article ID 927279, 2012.

[2] O. A. Jaffery, I. Akhtar, S. M. Imran Majeed, and T. Zaidi, "Computational modeling of inertial effects on electromechanics of the heart," Journal of Computational and Nonlinear Dynamics, vol. 14, no. 10, Article ID 101006, 2019.

[3] M. P. Nash and P. J. Hunter, "Computational mechanics of the heart," Journal of elasticity and the physical science of solids, vol. 61, no. 1, pp. 113-141, 2000.

[4] S. Morimoto, Q. W. Lu, K. Harada et al., "Ca2+-desensitizing effect of a deletion mutation $\Delta \mathrm{K} 210$ in cardiac troponin $\mathrm{T}$ that causes familial dilated cardiomyopathy," Proceedings of the National Academy of Sciences, vol. 99, no. 2, pp. 913-918, 2002.

[5] Y. Usson, F. Parazza, P. S. Jouk, and G. Michalowicz, "Method for the study of the three dimensional orientation of the nuclei of myocardial cells in fetal human heart by means of confocal scanning laser microscopy," Journal of Microscopy, vol. 174, no. 2, pp. 101-110, 1994.

[6] T. O'Hara, L. Virag, A. Varro, and Y. Rudy, "Simulation of the undiseased human cardiac ventricular action potential: model formulation and experim ental validation," PLoS Computational Biology, vol. 7, no. 5, Article ID e1002061, 2011.

[7] P. Lafortune, R. Aris, M. Vazquez, and G. Houzeaux, "Coupled electromechanical model of the heart: parallel finite element formulation," International journal for numerical methods in biomedical engineering, vol. 28, no. 1, pp. 72-86, 2012.

[8] G. A. Holzapfel and R. W. Ogden, "Constitutive modelling of passive myocardium: a structurally based framework for material cha/racterization," Philosophical Transactions of the Royal Society A: Mathematical, Physical \& Engineering Sciences, vol. 367, no. 1902, pp. 3445-3475, 2009.

[9] A. Agianniotis, R. Rezakhaniha, and N. Stergiopulos, "A structural constitutive model considering angular dispersion and waviness of collagen fibres of rabbit facial veins," BioMedical Engineering Online, vol. 10, no. 1, pp. 18-27, 2011.

[10] C. J. Cyron and J. D. Humphrey, "Preferred fiber orientations in healthy arteries and veins understood from netting analysis," Mathematics and Mechanics of Solids, vol. 20, no. 6, pp. 680-696, 2015. 
[11] R. R. Baier, A. Gizzi, A. Loppini, C. Cherubini, and S. Filippi, "Modélisation des effets thermo-électro-mécaniques dans le tissu cardiaque orthotrope," Communications in Computational Physics, vol. 27, pp. 87-115, 2020, https://arxiv.org/abs/ 1805.00757.

[12] I. J. LeGrice, B. H. Smaill, L. Z. Chai, S. G. Edgar, J. B. Gavin, and P. J. Hunter, "Laminar structure of the heart: ventricular myocyte arrangement and connective tissue architecture in the dog," American Journal of Physiology - Heart and Circulatory Physiology, vol. 269, no. 2, pp. H571-H582, 1995.

[13] W. Daenen, F. Lacour-Gayet, T. Aberg et al., "Optimal structure of a congenital heart surgery department in durope," EACTS Congenital Heart Disease Committee, vol. 10, no. 8, pp. 343-351, 2003.

[14] S. Ghonim, I. Voges, P. D. Gatehouse et al., "Myocardial architecture, mechanics, and fibrosis in congenital heart disease," Frontiers in cardiovascular medicine, vol. 8, no. 4, pp. 30-34, 2017.

[15] W. Kroon, T. Delhaas, T. Arts, and P. Bovendeerd, "Computational modeling of volumetric soft tissue growth: application to the cardiac left ventricle," Biomechanics and Modeling in Mechanobiology, vol. 8, no. 4, pp. 301-309, 2009.

[16] M. Brida and M. A. Gatzoulis, "Adult congenital heart disease: past, present and future," Acta Paediatrica, vol. 108, no. 10, pp. 1757-1764, 2019.

[17] G. B. Sands, D. A. Gerneke, D. A. Hooks, C. R. Green, B. H. Smaill, and I. J. Legrice, "Automated imaging of extended tissue volumes using confocal microscopy," Microscopy Research and Technique, vol. 67, no. 5, pp. 227-239, 2005.

[18] E. K. Rodriguez, A. Hoger, and A. D. McCulloch, "Stressdependent finite growth in soft elastic tissues," Journal of Biomechanics, vol. 27, no. 4, pp. 455-467, 1994.

[19] T. F. Robinson, M. A. Geraci, E. H. Sonnenblick, and S. M. Factor, "Coiled perimysial fibers of papillary muscle in rat heart: morphology, distribution, and changes in configuration," Circulation Research, vol. 63, pp. 577-592, 1988.

[20] D. H. S. Lin and F. C. P. Yin, "A multiaxial constitutive law for mammalian left ventricular myocardium in steady-state barium contracture or tetanus," Journal of Biomechanical Engineering, vol. 120, no. 4, pp. 504-517, 1995.

[21] S. Carasso and H. Yang, A. Woo, M. A. Vannan, M. Jamorski, E. D. Wigle, H. Rakowski, Systolic myocardial mechanics in hypertrophic cardiomyopathy: novel concepts and implications for clinical status," Journal of the American Society of Echocardiography, vol. 21, pp. 675-683, 2008.

[22] T. Kashimura, M. Kodama, and Y. Aizawa, "Left ventricular pressure-volume loops during mechanical alternans in a patient with dilated cardiomyopathy," Heart, vol. 93, no. 2, Article ID 151, 2007.

[23] J. B. Caulfield and T. K. Borg, "The collagen network of the heart," Laboratory investigation; a journal of technical methods and pathology, vol. 40, no. 3, pp. 364-372, 1979. 